\title{
Life on the Treadmill
}

\author{
Krisztián Flautner
}

ARM Ltd

\begin{abstract}
Silicon technology evolution over the last four decades has yielded an exponential increase in integration densities with steady improvements of performance and power consumption at each technology generation. This steady progress has created a sense of entitlement for the riches that future process generations would bring. Today, however, classical process scaling seems to be dead and living up to technology expectations requires continuous innovation at many levels, which comes at steadily progressing implementation and design costs. Solutions to problems need to cut across layers of abstractions and require coordination between software, architecture and circuit features.
\end{abstract}

